\title{
Measuring reproductive tourism through an analysis of Indian ART clinic Websites
}

This article was published in the following Dove Press journal:

International Journal of General Medicine

17 September 2012

Number of times this article has been viewed

\section{Raywat Deonandan \\ Mirhad Loncar \\ Prinon Rahman \\ Sabrina Omar}

Interdisciplinary School of Health Sciences, University of Ottawa,

Ottawa, Canada
Correspondence: Raywat Deonandan Interdisciplinary School of Health Sciences, University of Ottawa, Ottawa, Ontario, Canada KIN 6XI Tel + I 6135625800 ext 8377 Fax +l 6I35625632

Email raywat.deonandan@uottawa.ca
Objectives: India is fast becoming the most prominent player in the global industry of reproductive tourism, in which infertile people cross international borders to seek assisted reproduction technologies. This study was conducted to better understand the extent and manner in which Indian clinics seek foreign clients.

Methods: A systematic search of official Indian assisted reproduction technologies clinic Websites was undertaken, and instances noted where foreign clients were overtly targeted, and where maternal surrogacy was overtly offered.

Results: A total of 159 clinics with Web addresses were identified, though only 78 had functioning Websites. All were published in English, with the majority clustered in the states of Maharashtra (14) and Gujarat (9). Of the 78 functioning Websites, 53 (68\%) featured some mention of maternal surrogacy services, and 42 (54\%) made overt overtures to foreign clients. Qualitative appeals to foreigners included instructions for international adoption, visa application, and the legal parental disposition of the surrogate. All Maharashtran clinic Websites that mentioned surrogacy also overtly featured reproductive tourism. Preimplantation diagnosis services were not offered disproportionately by clinics mentioning reproductive tourism.

Conclusions: Based upon clinic online profiles, reproductive tourism comprises a substantial fraction of India's assisted reproduction technologies clinics' business focus, clustering around its most tourist-friendly locales, and surrogacy may be a strong motivator for international clientele.

Keywords: assisted reproduction, global health, social media, marketing, development, IVF, surrogacy, PGD

\section{Introduction}

Reproductive medical tourism (RT), also known as "cross border reproductive care,"1 "reproductive exile,"2 and a host of other names, is the phenomenon whereby individuals cross international borders to seek and receive assisted reproductive technologies (ARTs). ${ }^{3}$ Reasons for such travel can include a lack of availability of the required services in the home country, and more affordable services being offered in the destination country. The latter factor is believed to be one of the primary drivers in the evolution of the Indian RT market.

In India, RT is but one player in a multibillion dollar panoply of well-developed medical tourism services; RT is thought to be worth up to half a billion US dollars annually. ${ }^{4}$ Services offered by Indian clinics run the full gamut of state-of-the-art ARTs, including gamete donation, intrauterine insemination, in vitro fertilization, intracytoplasmic sperm injection, preimplantation genetic diagnosis (PGD) and maternal surrogacy. 
It is the latter service that sets India apart from its competitors in the West, given the very large number of economically depressed, fertile young women who can be called upon as potential surrogate mothers. As well, PGD may be an emerging service that might disproportionately attract foreign clientele.

The clientele of Indian fertility clinics is largely unknown, since there are no published studies of Indian patient demographics. However, it is thought that in addition to clients from India's own growing middle class, foreign clients make up a substantial component of clinic clientele. This assumption is supported by a plethora of anecdotal and lay evidence, as well as the marketing profiles of the clinics themselves. Browsing the Websites of several Indian clinics will reveal repeated instances where services for foreigners are overtly mentioned. ${ }^{5}$

The purpose of the present study was to systematically elucidate the Indian RT clientele by examining the nature of Indian clinics' online marketing presence. By searching for and reviewing Indian ART clinics with Websites, we aimed to describe the ways in which clinics use this medium to advertise to non-Indian potential clients, and to determine the proportion of clinics that overtly service foreigners, particularly with respect to maternal surrogacy.

\section{Methods}

A Google search was performed using various combinations, abbreviations, and spellings of the following keywords: India, assisted reproduction, artificial reproduction, in vitro fertilization, intracytoplasmic sperm injection, infertility, fertility, ART clinic, IVF clinic, infertility centre/center, IVF India, reproductive services, and surrogacy. The names of Indian states or union territories were coupled with these keywords during Web searches so that a wide representation of clinics across the country could be obtained. Links from each hit were also explored. Each resulting site was visually and qualitatively explored to determine whether it was the official site of an India-based fertility clinic. Websites for branch offices outside of India were disallowed. Clinics that exist as part of a general hospital were allowed, provided that they had their own dedicated Website. The language of the Website was not a barrier to inclusion in this study.

All resulting sites were reviewed to reveal the following characteristics about each clinic: the specific reproductive services offered, location, whether maternal surrogacy is overtly mentioned, some measurement of the size of the clinic (in terms of its scale of operation), and whether cost and success rates are mentioned. Special attention was given to clinics in the states of Maharashtra and Andhra Pradesh, known respectively as top foreign and domestic tourist destinations. ${ }^{6}$ We defined an "overt" mention of surrogacy as any use of the word "surrogate" or "surrogacy." However, a distinction was made between those Websites that made it clear that surrogacy was an option for clients of that clinic, and those that simply mentioned surrogacy as being a popular aspect of assisted reproduction. This was assessed by qualitatively reading the text presented in each site.

\section{Results}

A total of 159 Websites seemingly representing Indian ART clinics were identified. Of those, 148 were found to be unique clinics, and 11 were branch offices or separate service Websites for the clinics already captured. Of the 148, only 78 sites were functional when accessed. While the clinics appear to be scattered all over the country, they cluster most prominently in the states of Maharashtra (35 URLs identified, 14 functioning) and Gujarat (21 identified, 9 functioning). The names, URLs, and locations of identified clinics with functioning Websites are listed in Table 1.

The costs, size, and services mentioned by each Website are summarized in Table 2 (with clinic ID numbers corresponding to those in Table 1), and the abbreviations used to describe services in Table 2 are explained in Table 3. Three possible conditions for maternal surrogacy were noted in Table 2: no mention (indicated by "NO"), a mention of the existence of surrogacy (indicated by "YES [A]"), and an overt mention of surrogacy as a treatment option offered at that clinic (indicated by "YES [B]").

The presence or absence of surrogacy or RT services is summarized in Figure 1. The number of clinics that overtly claimed to offer surrogacy services was 37 (47\%), while those having a passing mention of such services was $16(21 \%)$, for a combined percentage of $68 \%$ of clinics whose Websites featured at least some aspect of maternal surrogacy. Interestingly, 57 Websites (86\%) included some reference to RT services. Of those, 20 (26\%) sites made passing mention, in a variety of contexts, of medical or RT; $20(26 \%)$ featured mention of RT via patient testimonials or success stories; and 42 (54\%) overtly featured vacation or travel packages in coordination with fertility services, or made other overtures to foreign clientele, such as providing instructions for international adoption, visa application, and the legal parental disposition of the surrogate, as it applies in international contexts.

Additionally, 26 clinics (33\%) advertised PGD services on their Website. Of these, only three (21\%) were in the 


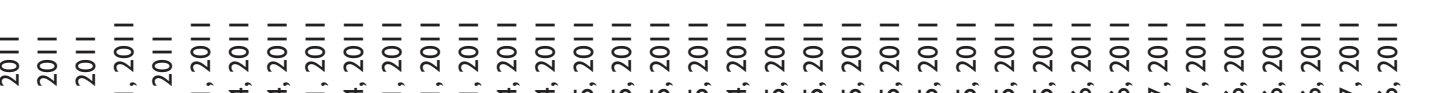

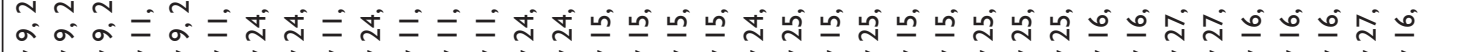

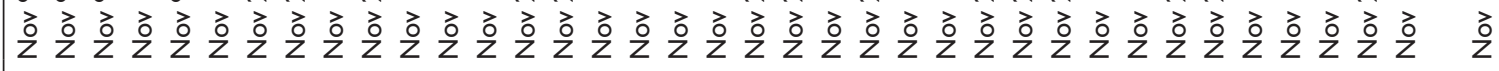
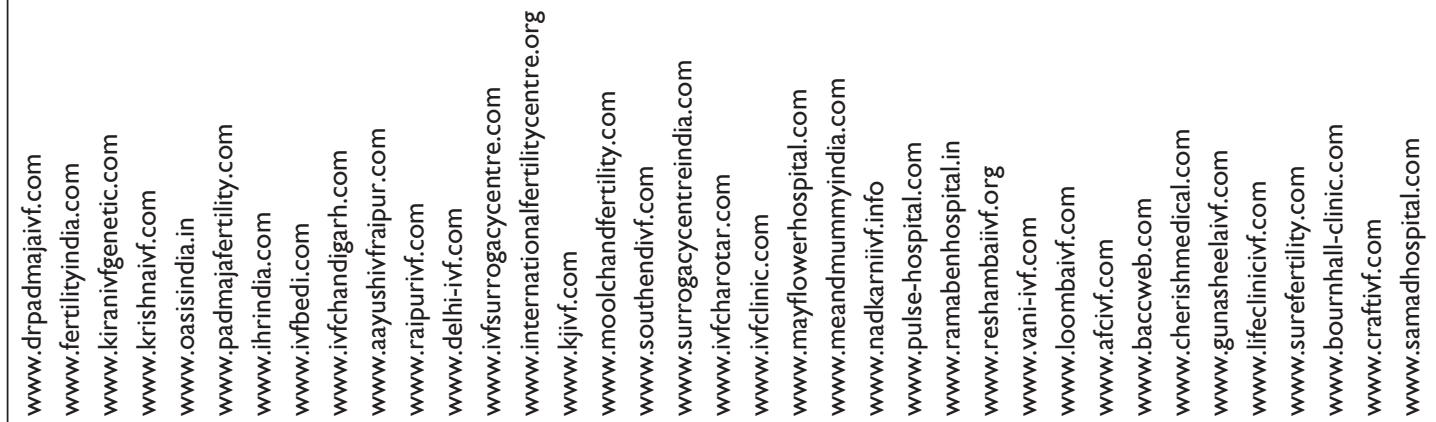

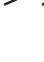

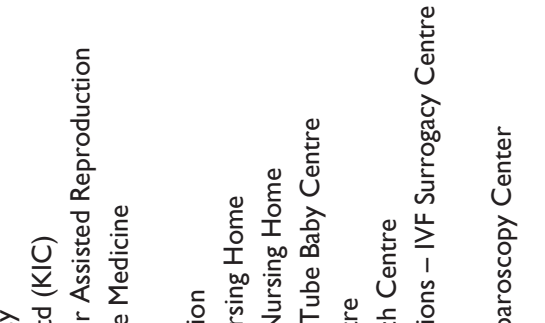



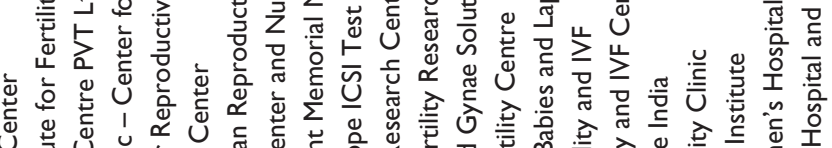

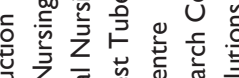

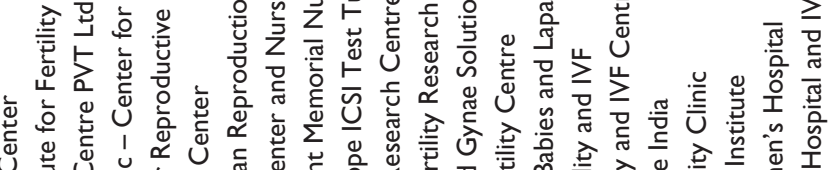

U⿺辶寸

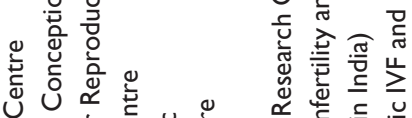
O. 


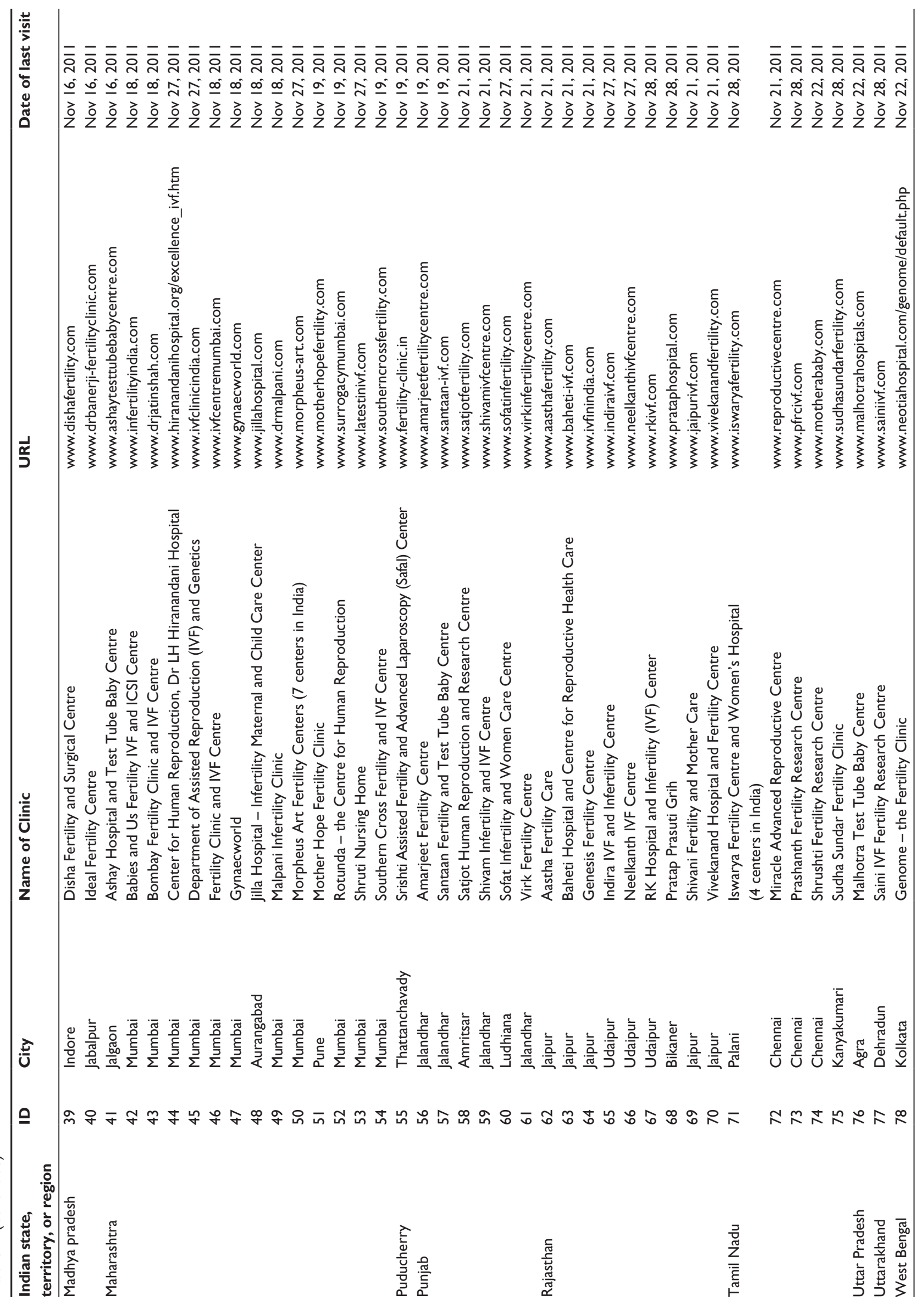


Table 2 Services advertised on each clinic's Website (see legend below)

\begin{tabular}{|c|c|c|c|}
\hline $\begin{array}{l}\text { Clinic ID } \\
\text { (see Table I) }\end{array}$ & $\begin{array}{l}\text { Services offered } \\
\text { (see Table } 3 \text { for explanation of codes) }\end{array}$ & $\begin{array}{l}\text { Surrogacy services } \\
\text { offered* }\end{array}$ & $\begin{array}{l}\text { Other } \\
\text { remarks** }\end{array}$ \\
\hline I & IVF, IUI, ICSI, AH, GD/ED, BC & Yes [B] & $\begin{array}{l}\text { RT: } 0 \\
\text { Size: } 3 \\
\text { Cost: } 0\end{array}$ \\
\hline 2 & IUI, IVF, ICSI, GIFT, SSR, CP, GD/ED, LAH, BC, CCC, PGD & Yes [B] & $\begin{array}{l}\text { RT: } 2,3 \\
\text { Size: } 3 \\
\text { Cost: } 0\end{array}$ \\
\hline 3 & IVF, IUI, ICSI, BC, GD, CP, SSR, ES & Yes [B] & $\begin{array}{l}\text { RT: } 2 \\
\text { Size: I, } 3 \\
\text { Cost: } 0\end{array}$ \\
\hline 4 & IUI, IVF, ICSI, AH, CP, SSR, PGD & No & $\begin{array}{l}\text { RT: } 3 \\
\text { Size: I, } 3 \\
\text { Cost: } 0\end{array}$ \\
\hline 5 & IVF, IUI, ICSI, ET, SSR, CP, AH, IVM & Yes $[\mathrm{B}]$ & $\begin{array}{l}\text { RT: } 3 \\
\text { Size: } 3 \\
\text { Cost: I }\end{array}$ \\
\hline 6 & IUI, IVF, ICSI, ET, CP, GD/ED, SSR, LAH, BC, CCC, PGD, ES & Yes [B] & $\begin{array}{l}\text { RT: } 0 \\
\text { Size: } 3 \\
\text { Cost: } 0\end{array}$ \\
\hline 7 & IUI, IVF, ICSI, ET, SSR, GIFT, CP, LAH, GD, PGD & No & $\begin{array}{l}\text { RT: } 3 \\
\text { Size: } 3 \\
\text { Cost: I }\end{array}$ \\
\hline 8 & IUI, IVF, ICSI, ET, SSR, GIFT, ZIFT, CP, GD/ED & No & $\begin{array}{l}\text { RT: I } \\
\text { Size: } 3 \\
\text { Cost: I }\end{array}$ \\
\hline 9 & IUI, IVF, ICSI, CP, SSR, AH, BC & No & $\begin{array}{l}\text { RT: I, } 3 \\
\text { Size: } 2,3 \\
\text { Cost: I }\end{array}$ \\
\hline 10 & IUI, IVF, ICSI, SSR, CP, BT, GD/ED, AH, ES & Yes [B] & $\begin{array}{l}\text { RT: } 0 \\
\text { Size: } 3 \\
\text { Cost: I }\end{array}$ \\
\hline II & IUI, IVF, ICSI, CP, EF, GD, ES & Yes [B] & $\begin{array}{l}\text { RT: } 3 \\
\text { Size: } 3 \\
\text { Cost: I }\end{array}$ \\
\hline 12 & IUI, IVF, ICSI, PGD, CP, BC, LAH, GD/ED, FET & Yes $[\mathrm{B}]$ & $\begin{array}{l}\text { RT: I, } 2 \\
\text { Size: I, } 3 \\
\text { Cost: I }\end{array}$ \\
\hline 13 & IUI, IVF, ICSI, BC, AH, CP, GD, SSR & Yes [B] & $\begin{array}{l}\text { RT: I } \\
\text { Size: } 2,3 \\
\text { Cost: I }\end{array}$ \\
\hline 14 & IUI, IVF, ICSI, GD, LAH, CP, BT, PGD & Yes [B] & $\begin{array}{l}\text { RT: } 3 \\
\text { Size: } 3 \\
\text { Cost: I }\end{array}$ \\
\hline 15 & IUI, IVF, ICSI, SSR, GIFT, GD/ED, CP, ES & Yes $[A]$ & $\begin{array}{l}\text { RT: } 3 \\
\text { Size: } 3 \\
\text { Cost: I }\end{array}$ \\
\hline 16 & IUI, IVF, ICSI, IVM, GD, IMCI, FERC, BC, BT, LAH, SSR, PGD & Yes [B] & $\begin{array}{l}\text { RT: } 2,3 \\
\text { Size: } 3 \\
\text { Cost: } 0\end{array}$ \\
\hline 17 & IUI, IVF, ICSI, ET, GIFT, CP, SSR, GD/ED & Yes [B] & $\begin{array}{l}\text { RT: I, } 2 \\
\text { Size: } 3 \\
\text { Cost: } 0\end{array}$ \\
\hline 18 & IUI, IVF, IVM, ICSI, SSR, LAH, BC, GD/ED, CP, PGD & Yes [B] & $\begin{array}{l}\text { RT: 2, } 3 \\
\text { Size: } 2,3 \\
\text { Cost: I }\end{array}$ \\
\hline
\end{tabular}


Table 2 (Continued)

\begin{tabular}{|c|c|c|c|}
\hline $\begin{array}{l}\text { Clinic ID } \\
\text { (see Table I) }\end{array}$ & $\begin{array}{l}\text { Services offered } \\
\text { (see Table } 3 \text { for explanation of codes) }\end{array}$ & $\begin{array}{l}\text { Surrogacy services } \\
\text { offered* }\end{array}$ & $\begin{array}{l}\text { Other } \\
\text { remarks** }\end{array}$ \\
\hline \multirow[t]{3}{*}{19} & IUI, IVF, ICSI, LAH, CP, PGD & Yes [B] & RT: 2,3 \\
\hline & & & Size: 2,3 \\
\hline & & & Cost: 0 \\
\hline \multirow[t]{3}{*}{20} & IUI, IVF, ICSI, SSR, BC, LAH, GD/ED, CP, PGD, ES & Yes [B] & RT: 2,3 \\
\hline & & & Size: 3 \\
\hline & & & Cost: 0 \\
\hline \multirow[t]{3}{*}{21} & IUI, IVF, ICSI, ET, SSR, CP, PGD & No & RT: I, 3 \\
\hline & & & Size: 3 \\
\hline & & & Cost: 0 \\
\hline \multirow[t]{3}{*}{22} & IUI, IVF, ICSI, ET, BT, GD, AH, SSR, GIFT, FERC & Yes $[\mathrm{A}]$ & RT: 0 \\
\hline & & & Size: 3 \\
\hline & & & Cost: 0 \\
\hline \multirow[t]{3}{*}{23} & IUI, IVF, ICSI, GIFT, ZIFT, SSR, GD/ED, CP, AH, BC & Yes $[\mathrm{A}]$ & RT: 3 \\
\hline & & & Size: 2,3 \\
\hline & & & Cost: 1 \\
\hline \multirow[t]{3}{*}{24} & IVF, ICSI, GD, CP, SSR, LAH, BC & Yes $[\mathrm{B}]$ & RT: 2,3 \\
\hline & & & Size: 2,3 \\
\hline & & & Cost: 1 \\
\hline \multirow[t]{3}{*}{25} & IUI, IVF, ICSI, CP, SSR & No & RT: 0 \\
\hline & & & Size: 3 \\
\hline & & & Cost: 0 \\
\hline \multirow[t]{3}{*}{26} & IUI, IVF, ICSI, SSR, AH, CP, GD/ED & Yes $[\mathrm{A}]$ & RT: 0 \\
\hline & & & Size: 3 \\
\hline & & & Cost: 0 \\
\hline \multirow[t]{3}{*}{27} & IUI, IVF, ICSI, SSR, CP, GD, PGD & Yes $[\mathrm{B}]$ & RT: 0 \\
\hline & & & Size: 3 \\
\hline & & & Cost: 1 \\
\hline \multirow[t]{3}{*}{28} & IUI, IVF, ICSI, SSR, CP, GD, PGD & Yes $[\mathrm{A}]$ & RT: I \\
\hline & & & Size: 2,3 \\
\hline & & & Cost: I \\
\hline \multirow[t]{3}{*}{29} & IUI, IVF, ICSI, ET, SSR, IVM, BC/BT, AH, CP & No & RT: 0 \\
\hline & & & Size: 3 \\
\hline & & & Cost: 0 \\
\hline \multirow[t]{3}{*}{30} & IUI, IVF, ICSI, ET, SSR LAH, BT, GD/ED, CP, GIFT, PGD & Yes $[\mathrm{A}]$ & RT: I \\
\hline & & & Size: I, 2, 3 \\
\hline & & & Cost: 0 \\
\hline \multirow[t]{3}{*}{31} & IUI, IVF, ICSI, SSR, GD/ED, BC, LAH, CP & No & RT: 0 \\
\hline & & & Size: 2,3 \\
\hline & & & Cost: 0 \\
\hline \multirow[t]{3}{*}{32} & IUI, IVF, IVM, ICSI, ET, SSR, BT, FET, LAH, GD/ED & No & RT: I \\
\hline & & & Size: 3 \\
\hline & & & Cost: 0 \\
\hline \multirow[t]{3}{*}{33} & IUI, IVF, ICSI, IVM, ET, CP, FET, GD, & No & RT: I, 2 \\
\hline & & & Size: 3 \\
\hline & & & Cost: 0 \\
\hline \multirow[t]{3}{*}{34} & IUI, IVF, ICSI, FET, CP, SSR & No & RT: 0 \\
\hline & & & Size: 3 \\
\hline & & & Cost: 1 \\
\hline \multirow[t]{3}{*}{35} & IUI, IVF, ICSI, CP, FET, GD/ED, ES & Yes $[\mathrm{A}]$ & RT: I, 3 \\
\hline & & & Size: 3 \\
\hline & & & Cost: 0 \\
\hline \multirow[t]{3}{*}{36} & IUI, IVF, IVM, ICSI, SSR, FET, AH, PGD & No & RT: 2,3 \\
\hline & & & Size: I, 2, 3 \\
\hline & & & Cost: 1 \\
\hline 37 & IUI, IVF, ICSI, ET, BT, SSR, LAH, GD & Yes $[\mathrm{A}]$ & RT: 3 \\
\hline & & & Size: 3 \\
\hline & & & Cost: I \\
\hline
\end{tabular}


Table 2 (Continued)

\begin{tabular}{|c|c|c|c|}
\hline $\begin{array}{l}\text { Clinic ID } \\
\text { (see Table I) }\end{array}$ & $\begin{array}{l}\text { Services offered } \\
\text { (see Table } 3 \text { for explanation of codes) }\end{array}$ & $\begin{array}{l}\text { Surrogacy services } \\
\text { offered* }\end{array}$ & $\begin{array}{l}\text { Other } \\
\text { remarks** }\end{array}$ \\
\hline 38 & IUI, IVF, ICSI, SSR, CP & No & $\begin{array}{l}\text { RT: } 0 \\
\text { Size: I, 2, } 3 \\
\text { Cost: } 0\end{array}$ \\
\hline 39 & IUI, IVF, ICSI, SSR, GD/ED, CP & Yes $[A]$ & $\begin{array}{l}\text { RT: } 0 \\
\text { Size: }[\mathrm{n} / \mathrm{a}] \\
\text { Cost: } 0\end{array}$ \\
\hline 40 & IUI, IVF, ICSI, SSR, GD, CP, BT & No & $\begin{array}{l}\text { RT: } 3 \\
\text { Size: } 3 \\
\text { Cost: I }\end{array}$ \\
\hline $4 I$ & IUI, IVF, ICSI, AH, GD/ED, SSR, CP, BT, PGD, ES & Yes $[A]$ & $\begin{array}{l}\text { RT: I, } 3 \\
\text { Size: } 3 \\
\text { Cost: } 0\end{array}$ \\
\hline 42 & IUI, IVF, ICSI, GD/ED, LAH, BC, SSR, CP, PGD, ES & No & $\begin{array}{l}\text { RT: I } \\
\text { Size: } 3 \\
\text { Cost: } 0\end{array}$ \\
\hline 43 & IUI, IVF, ICSI, ET, BT, GD/ED & Yes [B] & $\begin{array}{l}\text { RT: } 3 \\
\text { Size: I, } 3 \\
\text { Cost: } 0\end{array}$ \\
\hline 44 & IUI, IVF, ICSI, SSR, AH, BT, CP, GD/ED, ES & Yes [B] & $\begin{array}{l}\text { RT: } 2,3 \\
\text { Size: } 3 \\
\text { Cost: I }\end{array}$ \\
\hline 45 & IUI, IVF, ICSI, SSR, CAT, LAH, BC, CP, GD/ED, PGD & Yes $[A]$ & $\begin{array}{l}\text { RT: } 3 \\
\text { Size: } 3 \\
\text { Cost: } 0\end{array}$ \\
\hline 46 & IUI, IVF, ICSI, ET, SSR, LAH, BC, CP, GD/ED, & Yes [B] & $\begin{array}{l}\text { RT: } 2,3 \\
\text { Size: } 3 \\
\text { Cost: } 0\end{array}$ \\
\hline 47 & IUI, IVF, ICSI, SSR, FET, CP, GD/ED, & Yes $[\mathrm{B}]$ & $\begin{array}{l}\text { RT: } 3 \\
\text { Size: } 3 \\
\text { Cost: } 0\end{array}$ \\
\hline 48 & IUI, IVF, ICSI, SSR, CP, GD & Yes $[A]$ & $\begin{array}{l}\text { RT: I, } 3 \\
\text { Size: I, } 3 \\
\text { Cost: I }\end{array}$ \\
\hline 49 & IUI, IVF, ICSI, SSR, PGD, CP, LAH, GD/ED, BT & Yes [B] & $\begin{array}{l}\text { RT: } 2,3 \\
\text { Size: [n/a] } \\
\text { Cost: I }\end{array}$ \\
\hline 50 & IUI, IVF, ICSI, SSR, CP, GD & Yes $[A]$ & $\begin{array}{l}\text { RT: I, } 3 \\
\text { Size: I, } 3 \\
\text { Cost: I }\end{array}$ \\
\hline 51 & IUI, IVF, ICSI, SSR, BT, CP, GD/ED & No & $\begin{array}{l}\text { RT: } 0 \\
\text { Size: } 2,3 \\
\text { Cost: } 0\end{array}$ \\
\hline 52 & IUI, IVF, ICSI, ET, SSR, BT, BET, CP, LAH, GD/ED, & Yes [B] & $\begin{array}{l}\text { RT: } 2,3 \\
\text { Size: } 3 \\
\text { Cost: } 0\end{array}$ \\
\hline 53 & IUI, IVF, ICSI, ET, BT, AH, GD/ED, CP, PGD & No & $\begin{array}{l}\text { RT: } 0 \\
\text { Size: } 3 \\
\text { Cost: I }\end{array}$ \\
\hline 54 & IVF, ICSI, GIFT, ZIFT, TET, FET, AH, CP, BT & No & $\begin{array}{l}\text { RT: } 0 \\
\text { Size: } 2,3 \\
\text { Cost: } 0\end{array}$ \\
\hline 55 & IUI, IVF, IVM, ICSI, IMSI, SSR, FERC, GD/ED, CP, LAH, BC & No & $\begin{array}{l}\text { RT: I } \\
\text { Size: } 3 \\
\text { Cost: } 0\end{array}$ \\
\hline 56 & IUI, IVF, ICSI, GIFT, LAH, GD/ED & No & $\begin{array}{l}\text { RT: } 0 \\
\text { Size: } 3 \\
\text { Cost: } 0\end{array}$ \\
\hline
\end{tabular}


Table 2 (Continued)

\begin{tabular}{|c|c|c|c|}
\hline $\begin{array}{l}\text { Clinic ID } \\
\text { (see Table I) }\end{array}$ & $\begin{array}{l}\text { Services offered } \\
\text { (see Table } 3 \text { for explanation of codes) }\end{array}$ & $\begin{array}{l}\text { Surrogacy services } \\
\text { offered* }\end{array}$ & $\begin{array}{l}\text { Other } \\
\text { remarks** }\end{array}$ \\
\hline \multirow[t]{3}{*}{57} & IUI, IVF, ICSI, ET, GD/ED & No & RT: 0 \\
\hline & & & Size: 3 \\
\hline & & & Cost: 0 \\
\hline \multirow[t]{3}{*}{58} & IUI, IVF, ICSI, ET, SSR, CP, GD/ED, & Yes $[\mathrm{A}]$ & RT: 3 \\
\hline & & & Size: 3 \\
\hline & & & Cost: 0 \\
\hline \multirow[t]{3}{*}{59} & IUI, IVF, ICSI, SSR, CP, GIFT, ZIFT, GD/ED & No & RT: 3 \\
\hline & & & Size: 3 \\
\hline & & & Cost: 0 \\
\hline \multirow[t]{3}{*}{60} & IUI, IVF, ICSI, SSR, CP & No & RT: 0 \\
\hline & & & Size: 3 \\
\hline & & & Cost: 0 \\
\hline \multirow[t]{3}{*}{61} & IUI, IVF, ICSI, GIFT, ZIFT, SSR, AH & Yes [B] & $\mathrm{RT}: 2,3$ \\
\hline & & & Size: I, 3 \\
\hline & & & Cost: 1 \\
\hline \multirow[t]{3}{*}{62} & IUI, IVF, ICSI, SSR, FET, CP & No & RT: 0 \\
\hline & & & Size: 3 \\
\hline & & & Cost: I \\
\hline \multirow[t]{3}{*}{63} & IVF, ICSI, SSR, GD/ED, BT, CP & No & RT: I, 3 \\
\hline & & & Size: 3 \\
\hline & & & Cost: 1 \\
\hline \multirow[t]{3}{*}{64} & IUI, IVF, SSR, GD/ED, CP, AH, ES & Yes [B] & RT: I, 3 \\
\hline & & & Size: 3 \\
\hline & & & Cost: 0 \\
\hline \multirow[t]{3}{*}{65} & IUI, IVF, ICSI, SSR, LAH, CP, GD/ED, BC/BT, PGD & No & RT: I \\
\hline & & & Size: $[\mathrm{n} / \mathrm{a}]$ \\
\hline & & & Cost: I \\
\hline \multirow[t]{3}{*}{66} & IUI, IVF, IVM, ICSI, BC, CP, ES & Yes [B] & $\mathrm{RT}: 0$ \\
\hline & & & Size: 3 \\
\hline & & & Cost: 0 \\
\hline \multirow[t]{3}{*}{67} & IUI, IVF, ICSI, SSR, GD/ED, CP, BC & Yes [B] & RT: 0 \\
\hline & & & Size: 2,3 \\
\hline & & & Cost: 0 \\
\hline \multirow[t]{3}{*}{68} & IUI, IVF, ICSI, SSR, CP, BC, GD/ED, & Yes $[\mathrm{A}]$ & RT: I, 3 \\
\hline & & & Size: 3 \\
\hline & & & Cost: 0 \\
\hline \multirow[t]{3}{*}{69} & IUI, IVF, ICSI, ET, SSR, CP, PGD, ES & Yes [B] & $\mathrm{RT}: 3$ \\
\hline & & & Size: 3 \\
\hline & & & Cost: 0 \\
\hline \multirow[t]{3}{*}{70} & IUI, IVF, ICSI, SSR & No & $\mathrm{RT}: 3$ \\
\hline & & & Size: 3 \\
\hline & & & Cost: 0 \\
\hline \multirow[t]{3}{*}{71} & IUI, IVF, ICSI, ET, CP, BC, LAH, GD/ED, ES & Yes [B] & RT: 0 \\
\hline & & & Size: I, 2, 3 \\
\hline & & & Cost: 0 \\
\hline \multirow[t]{3}{*}{72} & IUI, IVF, ICSI, IVM, SSR, CP, BC, GD/ED, AH, PGD, ES & Yes [B] & RT: 3 \\
\hline & & & Size: 3 \\
\hline & & & Cost: I \\
\hline \multirow[t]{3}{*}{73} & IUI, IVF, ICSI, IMSI, AH, BT, CP, GD/ED, PGD & Yes [B] & RT: 2, 3 \\
\hline & & & Size: 2,3 \\
\hline & & & Cost: I \\
\hline \multirow[t]{3}{*}{74} & IUI, IVF, ICSI, ET, SSR, GD/ED, PGD & Yes [B] & RT: 2, 3 \\
\hline & & & Size: 2,3 \\
\hline & & & Cost: 0 \\
\hline 75 & IUI, IVF, ICSI, CP, PGD & No & RT: 0 \\
\hline & & & Size: $[\mathrm{n} / \mathrm{a}]$ \\
\hline & & & Cost: 0 \\
\hline
\end{tabular}


Table 2 (Continued)

\begin{tabular}{llll}
\hline $\begin{array}{l}\text { Clinic ID } \\
\text { (see Table I) }\end{array}$ & $\begin{array}{l}\text { Services offered } \\
\text { (see Table } 3 \text { for explanation of codes) }\end{array}$ & $\begin{array}{l}\text { Surrogacy services } \\
\text { offered* }\end{array}$ & $\begin{array}{l}\text { Other } \\
\text { remarks*** }\end{array}$ \\
\hline 76 & IUI, IVF, ICSI, SSR, CP, BC, GD/ED, AH, PGD & Yes [B] & $\begin{array}{l}\text { RT: I } \\
\text { Size: } 3 \\
\text { Cost: } 0\end{array}$ \\
77 & & RT: 3 & Size: 2,3 \\
& IUI, IVF, IVM, ICSI, ET, SSR, GD/ED, CP & Cost: 0 \\
78 & & Yes [B] & RT: 2, 3 \\
& IVF, ICSI, ET, SSR, CP, AH, GD/ED & Yes [A] & Size: I, 2, 3 \\
& & & Cost: 0 \\
\hline
\end{tabular}

Notes: *A = mention, $\mathrm{B}=$ overt claim or offer; $* * \mathrm{RT}$ : $0=$ no mention on Website, $\mathrm{I}=$ mentioned somewhere on Website (ie, reproductive services offered to non-Indian infertile couples), 2 = mention through advertising of patient feedback letters, success stories, or testimonials, $3=$ advertising of special treatment packages/services for overseas patients and/or suggestions for accommodation, transport, or sightseeing; Size: I = mention of the number of procedures done in a specified time period and/or the number of successful pregnancies, 2 = mention of the number of patients seen at clinic to date or in a specified time period, and/or the number of successful deliveries, 3 = mention of the members of the clinical staff; cost: $0=$ no indication of cost, 1 = cost of some or all services mentioned.

Abbreviation: RT, reproductive medical tourism.

high volume area of Maharashtra, and four (44\%) in Gujarat; whereas four of the five ( $80 \%)$ Tamil Nadu clinics, and three of the six (50\%) Andhra Pradesh clinics offered PGD.

Among the clinics advertising RT with overt overtures to foreigners, ten $(23 \%)$ were in the Maharashtra area, five $(12 \%)$ each were in the states of Gujarat and Rajasthan, four $(10 \%)$ were in New Delhi, and three $(7 \%)$ were in each of Andhra Pradesh and Tamil Nadu.

Table 3 Explanation of ART service codes used in Table 2

\begin{tabular}{ll}
\hline Service abbreviation & Actual service \\
\hline AH (LAH) & Assisted hatching (laser-assisted hatching) \\
BC & Blastocyst culture \\
BT & Blastocyst transfer \\
CAT & Cumulus-aided embryo transfer \\
CCC & Cumulus cell coculture \\
CP & Cryopreservation (includes sperm, \\
& embryo, and oocyte freezing) \\
ED & Embryo donation \\
ES & Egg sharing \\
ET & Embryo transfer \\
FERC & Frozen embryo replacement cycle \\
FET & Frozen embryo transfer \\
GD & Gamete donation \\
GIFT & Gamete intrafallopian transfer \\
ICSI & Intracytoplasmic sperm injection \\
IMSI & Intracytoplasmic morphologically-selected \\
& sperm injection \\
IUI & Intrauterine insemination \\
IVF & In vitro fertilization \\
IVF-ET & In vitro fertilization - embryo transfer \\
IVM & In vitro maturation \\
PGD & Preimplantation genetic diagnosis \\
TET & Tubal embryo transfer \\
SSR & Surgical sperm retrieval \\
ZIFT & Zygote intrafallopian transfer \\
\hline Abbreviation
\end{tabular}

Abbreviation: ART, assisted reproductive technologies.
Amongst the 14 clinics in Maharasthra, ten Websites mentioned surrogacy services and all ten $(100 \%)$ also overtly talked about RT opportunities. Amongst the six clinics in Andhra Pradesh, five (83\%) mentioned surrogacy services and four (67\%) mentioned RT.

\section{Discussion}

Our approach in this study was to examine solely the online presence of Indian ART clinics to gain a sense of the extent to which efforts were being made to attract foreign clientele. Our methods reflect those of several earlier writers in a variety of therapeutic areas. ${ }^{7-9}$ Almost universally, those writers found that online representations of clinical services suffered from imprecise or often misleading information, due in part to their lack of the most recent, peer-reviewed information.

The non-governmental organization Sama's report ${ }^{5}$ on the advertising techniques used by Indian fertility clinics was the first comprehensive overview of language, imagery,



Figure I The availability of maternal surrogacy and reproductive medical tourism (RT) services in Indian clinics, based upon examination of 78 Websites. 
and strategies used by ART providers to attract clientele to their clinics. It was suggested from their analysis that, within a purely capitalist milieu, the communication of risk and potential ethical missteps took a back seat to the generation of new business. They pointed to such questionable strategies as the publication of what they referred to as "milestones" (eg, implantation rates) in lieu of actual success rates (eg, rate of deliveries of healthy, live babies), as an effort to increase the perception of ART as being more successful than the objective science might otherwise suggest, an observation recapitulated in our earlier work. ${ }^{10}$

Given the heavily commercialized environment in which Indian ART clinics must operate, the temptation to present only that information most attractive to potential clientele is great indeed. The aforementioned use of milestones in lieu of success rate data is but one manifestation of this failing. Full transparency would require a listing of both milestones and success rates that are disaggregated according to underlying causes of infertility, ${ }^{11}$ as well as some mention of the risks associated with ART, such as multiple pregnancies and extensive use of pharmaceuticals. ${ }^{12}$

Unlike Sama's more exhaustive analysis of advertising strategies, ${ }^{5}$ we sought to examine the extent of RT in India solely through clinic online presence. To that end, it was not surprising that the state of Maharashtra, the nation's financial center, would prove to be the source of a plurality of clinics overtly offering tourism services or information alongside ART services. All but three of the 14 Maharasthran Websites advertised a tourism connection to their services, with a majority of clinics situated in Mumbai, known for its tourist infrastructure and globally-advertised cultural products, such as Bollywood movies.

India's top tourist destination for foreigners tend to be the states of Maharashtra (5.1 million foreigners in 2010), Tamil Nadu (2.8 million), the territory of Delhi (1.9 million), Uttar Pradesh (1.7 million), Rajasthan (1.3 million), and West Bengal (1.2 million). ${ }^{7}$ In terms of domestic tourism, Andhra Pradesh and Maharasthra were the top destinations in $2010 .^{7}$ Consequently, these areas were of particular interest to us.

Therefore, it was not surprising that three out of five Tamil Nadu clinics examined, four out of seven Delhi clinics, three out of four Keralan clinics, and the sole West Bengali clinic were among those showing an overt courting of foreign tourists seeking ART. These are high proportions, which suggest that there may be a relationship between those regions' foreign tourist traffic and their provision of ART services. Our focus on Maharashtra supports this supposition, as all ten of that state's clinics that mention surrogacy also advertise
RT, suggesting that surrogacy is one of the main attractors of the foreign clientele being sought.

Another suspected attractor was PGD, known to be only limitedly available in some countries, due to both its relative newness on the technological landscape, and its ethical issues. ${ }^{13}$ But only three clinics in Maharashtra offer PGD, whereas four out of five clinics in Tamil Nadu, and three out of six clinics in Andhra Pradesh offer it. This suggests that PGD may not be a factor motivating international RT. But, given the domestic tourist traffic to Tamil Nadu and Andhra Pradesh, it is possible that PGD might be perceived as a service to be marketed to Indians more than to foreigners.

Our findings are suggestive, but not conclusive. To date, barriers to the understanding of the motivators and extent of RT in India have been substantial. Lack of pooled national patient data and an understandable reluctance on the part of clinics to open their patient data to analysis of this sort, compel us to rely on proxy measurements of international service usage. It is not even known from which countries Indian RT clients typically arise, though India as a whole is most likely to be visited by tourists from the USA and UK. ${ }^{14}$ What we can say at this point is that, based upon our analyses of clinic online profiles, it seems that RT comprises a substantial fraction of India's ART business focus, clustering around its most tourist-friendly locales, and that surrogacy is a strong motivator for international clientele.

\section{Disclosure}

The authors report no conflicts of interest in this work.

\section{References}

1. Johnston R, Crooks VA, Snyder J, Kingsbury P. What is known about the effects of medical tourism in destination and departure countries? A scoping review. Int J Equity Health. 2010;9:24.

2. Ramírez de Arellano AB. Patients without borders: the emergence of medical tourism. Int J Health Serv. 2007;37(1):193-198.

3. Cortez N. Patients without borders: the emerging global market for patients and the evolution of modern health care. Indiana Law Journal. 2008;83:71-132.

4. Schiano TD, Rhodes R. The dilemma and reality of transplant tourism: an ethical perspective for liver transplant programs. Liver Transpl. 2010;16(2):113-117.

5. Sama (Resource Group for Women and Health). Cheap and Best: Analysis of Websites, Brochures and Advertisements on Assisted Reproductive Technologies in India. New Delhi: Impulsive Creations; 2008.

6. The Financial Express. Andhra Pradesh top tourist destination: Tourism Ministry. July 18, 2011. Available from: http://bit.ly/NxKibD. Accessed August 24, 2012.

7. Lagu T, Hannon NS, Rothberg MB, Lindenauer PK. Patients' evaluations of health care providers in the era of social networking: an analysis of physician-rating websites. J Gen Intern Med. 2010;25(9):942-946.

8. Maifredi G, Orizio G, Bressanelli M, et al. Italian hospitals on the web: a cross-sectional analysis of official websites. BMC Med Inform Decis Mak. 2010;10:17. 
9. Bucksch J, Kolip P, Deitermann B. Reporting on post-menopausal hormone therapy: an analysis of gynaecologists' web pages. Med Inform Internet Med. 2004;29(3-4):211-220.

10. Deonandan R, Campbell MK, Østbye T, Tummon I. Toward a more meaningful in vitro fertilization success rate. J Assist Reprod Genet. 2000;17(9):498-503.

11. Shanner L, Nisker J. Bioethics for clinicians: 26 . Assisted reproductive technologies. CMAJ. 2001;164(11):1589-1594.
12. Elster, N. Less is more: the risks of multiple births. The Institute for Science, Law, and Technology Working Group on Reproductive Medicine. Fertil Steril. 2000;74(4):617-623.

13. Mauron A. Ethical aspects of preimplantation genetic diagnosis (PGD). Bull Acad Natl Med. 2011;195(4-5):1023-1031. French.

14. The Economic Times. US tops foreign tourist arrivals in India. January 5, 2011. Available from: http://bit.ly/SWMqJn. Accessed August 24, 2012.

\section{Publish your work in this journal}

The International Journal of General Medicine is an international, peer-reviewed open-access journal that focuses on general and internal medicine, pathogenesis, epidemiology, diagnosis, monitoring and treatment protocols. The journal is characterized by the rapid reporting of reviews, original research and clinical studies across all disease areas.

\section{Dovepress}

A key focus is the elucidation of disease processes and management protocols resulting in improved outcomes for the patient.The manuscript management system is completely online and includes a very quick and fair peer-review system. Visit http://www.dovepress.com/ testimonials.php to read real quotes from published authors.

Submit your manuscript here: http://www.dovepress.com/international-journal-of-general-medicine-journal 